Vera Maria Gomes de Moura Sena ${ }^{1}$ Laura Olinda BreGieiro Fernandes Costa ${ }^{2}$ Hélio de Lima Ferreira Fernandes Costa ${ }^{3}$

Artigos originais

Palavras-chave

Menopausa/efeitos de drogas Isoflavonas/uso terapêutico

Fogachos

Endométrio/anatomia \& histologia

Keywords

Menopause/drug effects Isoflavones/therapeutic use

Hot flashes

Endometrium/anatomy \& histology

\section{Efeitos da isoflavona de soja sobre os sintomas climatéricos e espessura endometrial: ensaio clínico, randomizado duplo-cego e controlado}

\author{
Effects of soy-derived isoflavone on hot flushes and endometrial \\ thickness: a randomized, double-blind controlled trial
}

\title{
Resumo
}

OBJETIVO: avaliar o efeito da isoflavona de soja sobre os sintomas climatéricos e espessura endometrial em mulheres na pós-menopausa. MÉTODOS: ensaio clínico randomizado, duplo-cego e controlado envolvendo 90 mulheres pósmenopausadas, entre 45 e 60 anos, com queixas de fogachos, acompanhados ou não de outros sintomas climatéricos. Foram randomizadas para receber $50 \mathrm{mg}$ de isoflavona a cada 12 horas, diariamente, por 12 semanas (n=42), ou placebo ( $n=48$ ), e avaliadas antes e ao final do tratamento, pelo índice menopausal de Kupperman (IK), no qual se registrava a intensidade dos sintomas climatéricos, utilizando uma escala visual analógica de $100 \mathrm{~mm}$. Os fogachos foram avaliados separadamente também. Foram submetidas a uma ultra-sonografia transvaginal para a avaliação da espessura do eco endometrial. Para a análise estatística, empregaram-se o teste do $\chi^{2}$, ANOVA ou $t$ de Student e Mann-Whitney, ao nível de significância de 5\%. RESULTADOS: não foram observadas diferenças significantes no IK $(64$ versus $82, p>0,05)$ nem nos fogachos (20 versus $20, p>0,05)$, entre os grupos tratados com isoflavona ou placebo, respectivamente. Também não foram observadas diferenças significantes no IK e nos fogachos, antes e após o tratamento, quando os grupos tratados com isoflavona ou placebo foram analisados separadamente. Nenhuma diferença foi observada na espessura do endométrio após o tratamento entre os grupos tratados com isoflavona ou placebo 10,28 versus $0,26 \mathrm{~mm}$, respectivamente, p>0,05). CONCLUSÕES: a isoflavona de soja, na dose de $100 \mathrm{mg} /$ dia, não é mais efetiva que o placebo para a redução das ondas de calor e sintomas do hipoestrogenismo em mulheres na pósmenopausa e não apresenta efeitos sobre a espessura endometrial.

\section{Abstract}

PURPOSE: to determine the effects of soy-derived isoflavone on hot flashes, menopausal symptoms, and endometrial thickness in postmenopausal women. METHODS: this double-blind, placebo-controlled, randomized study involved 90 postmenopausal patients aged 45-60 years old attended at the Outpatient Menopause Clinic. All patients had been experiencing hot flashes accompanied or not by other hypo-estrogenic symptoms. Patients were randomized to receive either two soy capsules containing $50 \mathrm{mg}$ of soy-derived isoflavone or two identical placebo capsules, twice a day for 12 weeks in a double-blind fashion. Each patient was observed for 12 weeks, with two evaluations being made, one at baseline and the other at the end of the study. At each time point, the patients were given a diary to record the severity of the climacteric symptoms experienced, assessed with a modified Kupperman index, using a 100 mm Visual Analogue Scale (VAS). The intensity of hot flashes was also assessed separately. The patients were also submitted to a transvaginal echography for the measurement of endometrial thickness. Yates $\chi^{2}$, ANOVA or $t$ de Student and Mann-Whitney were used for statistical analysis. RESULTS: no significant difference was detected in the Kupperman index $(64$ versus $82, p>0,05)$ or in the hot flashes (20 versus $20, p>0,05$ ) between the isoflavone and placebo groups. No significant difference was either detected concerning the Kupperman index and hot flashes before and after treatment, when the two groups were analyzed separately. No difference was detected in the endometrial thickness either in the isoflavone or the placebo group $(0.28$ versus $0.26 \mathrm{~mm}$, respectively, p>0.05). CONCLUSIONS: our results indicate that $100 \mathrm{mg}$ of isoflavone are not more effective than placebo in reducing hot flashes and hypo-estrogenic symptoms in postmenopausal women and present no effect on the endometrium thickness.

Correspondência:

Laura Olinda Bregieiro Fernandes Costa Rua Bruno Maia, 217, apto. 1.001 - Graças CEP $51011-110-$ Recife/PE Fone: (81) 32212017 E-mail: heliolaur@yahoo.com.br

Recebido

$11 / 09 / 2007$

Aceito com modificacões

$29 / 10 / 2007$
Trabalho realizado no Departamento Materno-Infantil da Faculdade de Ciências Médicas da Universidade de Pernambuco - UPE Pernambuco (PE), Brasil.

'Médica Assistente do Hospital Barão de Lucena - Recife (PE), Brasil; Pós-graduanda do Departamento Materno-Infantil da Faculdade de Ciências Médicas da Universidade de Pernambuco - UPE - Recife (PE), Brasil.

2 Professora Adjunta do Departamento Materno-Infantil da Faculdade de Ciências Médicas da Universidade de Pernambuco - UPE Recife (PE), Brasil.

${ }^{3}$ Professor Regente da Disciplina de Tocoginecologia do Departamento Materno-Infantil da Faculdade de Ciências Médicas da Universidade de Pernambuco - UPE - Recife (PE), Brasil. 


\section{Introdução}

A estrogenioterapia é considerada o tratamento de escolha para o alívio dos sintomas relacionados ao hipoestrogenismo da mulher climatérica. Entretanto, estudos recentes têm atribuído à terapia estrogênica ou estroprogestativa aumento no risco de câncer de mama e tromboembolismo e, desta forma, seu uso tem sido limitado ${ }^{1}$. Por essa razão, acrescida ao fato da crescente demanda por alternativas terapêuticas naturais, um número significativo de mulheres climatéricas tem utilizado os fitoestrogênios, particularmente a isoflavona, como alternativa terapêutica para o alívio dos sintomas climatéricos ${ }^{2}$.

As isoflavonas são compostos não esteróides, estruturalmente similares ao estrogênio natural, por apresentarem um anel fenólico com um radical hidroxila no carbono 3, estrutura que lhe confere a capacidade de ligação seletiva, de alta afinidade aos receptores estrogênicos ${ }^{3}$. Possuem efeito estrogênico ou antiestrogênico, dependendo da concentração dos mesmos, da concentração dos esteróides sexuais endógenos e do órgão-alvo específico envolvido na interação com os receptores estrogênicos. A existência de dois tipos de receptores estrogênicos, alfa e beta, confere especificidade dos diferentes órgãos-alvo aos fitoestrogênios ${ }^{4}$. São habitualmente encontradas na soja e em vários outros tipos de frutas, vegetais, grãos e legumes.

Dados epidemiológicos de estudos japoneses mostram que a intensidade dos sintomas vasomotores é inversamente proporcional ao consumo de soja na dieta $^{5}$. Alguns estudos observacionais sugerem melhora dos sintomas de hipoestrogenismo, particularmente dos fogachos, com a suplementação dietética do extrato da soja em mulheres climatéricas, sem efeitos negativos no endométrio ${ }^{6}$. Entretanto, os ensaios clínicos controlados que avaliam o efeito da isoflavona nos sintomas vasomotores são contraditórios. A redução da freqüência das ondas de calor não foi demonstrada em uma metaanálise que envolveu 17 ensaios clínicos controlados que utilizaram o extrato de isoflavona em mulheres climatéricas ${ }^{7}$. Outros estudos controlados têm mostrado que o extrato de soja, na dose de 50 a $100 \mathrm{mg} / \mathrm{dia}$, parece ser seguro e uma alternativa terapêutica eficaz para a mulher na pós-menopausa com sintomas climatéricos moderados $^{8-10}$, sem alterar a espessura endometrial ${ }^{10-12}$. Outros autores, embora não tenham observado alterações na espessura endometrial, detectaram algum grau de atividade mitótica endometrial durante o uso da isoflavona em mulheres na pós-menopausa ${ }^{13}$.

Além disso, a grande variedade de compostos contendo a isoflavona, em diferentes doses, nos inúmeros estudos publicados, tem dificultado a interpretação dos resultados e as revisões sistemáticas ${ }^{14}$.

Portanto, faz-se necessária a realização de novos estudos que avaliem a eficácia e a segurança do uso da isoflavona pela mulher climatérica. Assim, o presente ensaio clínico visa avaliar os efeitos da isoflavona de soja sobre os sintomas climatéricos e espessura endometrial.

\section{Métodos}

Este ensaio clínico, randomizado, duplo-cego, controlado com placebo, envolveu 90 mulheres na pós-menopausa, com idade entre 45 e 60 anos, assistidas no Ambulatório de Climatério da Disciplina de Tocoginecologia da Universidade de Pernambuco (UPE), em Recife (PE), recrutadas no momento da consulta de rotina realizada naquele ambulatório. Inicialmente, todas as pacientes foram submetidas a anamnese dirigida e exame físico, obedecendo a todos os procedimentos da propedêutica climatérica, incluindo a colpocitologia oncótica, ultra-sonografia endovaginal, mamografia bilateral e perfil lipídico. Foram incluídas mulheres com queixas de fogachos, acompanhados ou não de outros sintomas clínicos de hipoestrogenismo, níveis plasmáticos de $\mathrm{FSH} \geq 40 \mathrm{U} / \mathrm{mL}$ e ausência de menstruação há pelo menos seis meses. Não foram incluídas mulheres sob terapia hormonal ou outro tipo de tratamento hormonal, dieta com soja e seus derivados há pelo menos dois meses antes do início do estudo. As que referiam história de câncer de mama, hiperplasia endometrial, sangramento uterino anormal não diagnosticado, doenças tromboembólicas, doenças hepáticas ou renais ou alcoolismo crônico foram excluídas. Todas as mulheres assinaram, após esclarecimentos, o termo de consentimento livre e esclarecido. O estudo foi previamente aprovado pela Comissão de Ética da UPE.

As pacientes foram distribuídas, de acordo com uma lista de números randômicos gerados em computador (programa Epi-Table do Epi-Info 6.04), em dois grupos: um com 42 pacientes que usaram a isoflavona de soja na dose de $100 \mathrm{mg}$ por dia (50 mg a cada 12 horas) sob a forma de cápsulas, e outro com 48 pacientes que usaram placebo (uma cápsula a cada 12 horas), em embalagens idênticas e identificadas por um único número de série, por 12 semanas. Em cada cápsula com $50 \mathrm{mg}$ de isoflavona havia entre 40 e $45 \%$ de genisteína, 40 e $45 \%$ de daidzeína e 10 e $20 \%$ de gliciteína, enquanto as cápsulas-placebo, idênticas na aparência às de isoflavona, continham celulose microcristalina. Ambas foram comercializadas pela Galena Química e Farmacêutica Ltda. 
Cada paciente foi acompanhada por um período de três meses, sendo feita uma avaliação basal e após três meses de tratamento. A cada avaliação, as pacientes eram analisadas em relação aos sintomas climatéricos e submetidas à ultra-sonografia transvaginal. Os sintomas climatéricos foram registrados e pontuados em todas as pacientes, pelo índice menopausal de Kupperman (IK) antes e após três meses de tratamento. Este índice representa a soma dos escores de 11 sinais e sintomas relacionados ao hipoestrogenismo (fogachos, parestesia, insônia, nervosismo, melancolia, vertigem, fraqueza, artralgia, cefaléia, palpitação e formigamento), graduados de 0 a 3 , se forem ausentes, leves, moderados ou intensos. Multiplicam-se por 2 a parestesia, a insônia e o nervosismo e por 4 os fogachos.

Para a obtenção dos escores, utilizou-se a escala visual analógica. Esta escala, usada originalmente para a avaliação da intensidade da dor, foi utilizada no estudo para avaliar a intensidade dos sintomas climatéricos. Apresentava-se à paciente uma linha graduada de $0 \mathrm{a}$ 10 , cuja extremidade esquerda correspondia à ausência de sintomas (0), e sinais e sintomas muito severos, à extremidade oposta (10). As pacientes foram instruídas a atribuir escores na escala visual analógica correspondentes à intensidade dos sintomas climatéricos, em cada uma das avaliações (basal e três meses após o tratamento). A intensidade dos fogachos também foi analisada separadamente, seguindo os mesmos critérios de análise do IK. Foi avaliada também a resposta terapêutica global por meio da auto-avaliação da paciente e categorizada em: melhora total, na ausência de sintomas residuais; melhora parcial, na permanência de sintomas residuais, considerado pela paciente como perfeitamente suportável; ou ausência de resposta, na permanência dos sintomas na mesma intensidade.

A ultra-sonografia transvaginal foi realizada para avaliar a espessura endometrial, sendo considerado o ponto de maior espessura, com os "calipers" posicionados entre a área hiperecogênica endometrial e o halo hipoecogênico circundante, incluindo os dois folhetos endometriais. Foi utilizado um equipamento Toshiba Power Vision 600 equipado com um transdutor endovaginal de $6,5 \mathrm{MHz}$.

O tamanho amostral foi calculado com bases nas modificações estimadas do IK, após 12 semanas de tratamento com a isoflavona. Para se detectarem diferenças de, no mínimo, 45 unidades neste índice, entre o grupo tratado com isoflavona e o placebo, com um poder de teste de $80 \%$ e um nível de significância de 0,05 para teste bicaudal, foi necessário um tamanho amostral de 43 pacientes em cada grupo. Contando com perda de $30 \%$, um tamanho amostral de 56 pacientes por grupo seria suficiente para detectar as diferenças esperadas, o que resultou num grupo total de 112 pacientes.

Os dados foram processados com o programa EpiInfo 2005, versão 3.3.2, do Centers for Disease Control and Prevention (CDC) da World Health Organization (WHO) e Excel 2002, da Microsoft. Os testes estatísticos, realizados no nível de significância de $5 \%$, foram teste do $\chi^{2}$ com correção de Yates, para análise de associação entre variáveis, e ANOVA ou $t$ de Student, para comparar médias de duas populações com base em amostras dessas populações. Para a comparação das medianas do IK e dos fogachos, foi utilizado o teste não paramétrico de Mann-Whitney. A análise estatística foi realizada apenas com as pacientes que concluíram o estudo.

Cento e vinte pacientes foram selecionadas para participar do estudo, das quais quatro foram excluídas, três não preencheram os critérios de inclusão e três recusaram-se a participar. Assim, 110 pacientes foram randomizadas para receberem isoflavona de soja (55 pacientes) ou placebo ( 55 pacientes). Seis pacientes interromperam o tratamento (quatro do grupo tratado com isoflavona de soja e dois do Grupo Placebo) e houve perda de seguimento em 14 pacientes (nove do grupo tratado com isoflavona de soja e cinco do Grupo Placebo). Desta forma, concluíram os três meses de tratamento 42 pacientes do grupo tratado com isoflavona e 48 pacientes que usaram o placebo.

\section{Resultados}

A Tabela 1 evidencia as características clínicas iniciais dos dois grupos de pacientes. Observamos que os grupos estudados eram homogêneos em relação à idade, à idade da menopausa, ao tempo de menopausa e ao índice de massa corpórea (IMC).

Ao analisarmos a redução do IK nos Grupos Isoflavona e Placebo (64 versus 82, respectivamente, $\mathrm{p}>0,05)$, não observamos diferença significante entre eles após os três meses de tratamento (Tabela 2). As reduções observadas nesse índice, antes e após o tratamento, tanto no grupo tratado com isoflavona (132 versus 64 , respectivamente, $\mathrm{p}>0,05$ ) como no Grupo Placebo (144 versus 82, respectivamente, $\mathrm{p}>0,05)$ também não foram significantes.

Ao analisarmos a variação dos escores atribuídos aos fogachos entre os Grupos Isoflavona e Placebo (20 versus 20 , respectivamente, $\mathrm{p}>0,05)$, não observamos diferença estatisticamente significante após os três meses de tratamento (Tabela 2). Também não foram encontradas reduções significantes nesses escores, antes e após o tratamento, tanto no grupo tratado com isoflavona (24 versus 20, respectivamente, $\mathrm{p}>0,05)$, como no Grupo Placebo (24 versus 20, respectivamente, $\mathrm{p}>0,05)$. 
Tabela 1 - Características clínicas das pacientes usuárias de isoflavonas e de placebo (média \pm desvio padrão).

\begin{tabular}{lccc}
\hline Variáveis & $\begin{array}{c}\text { Isoflavona } \\
(\mathbf{n}=42)\end{array}$ & $\begin{array}{r}\text { Placebo } \\
(\mathbf{n}=48)\end{array}$ & $p^{*}$ \\
\hline Idade (annos) & $52,5 \pm 6,7$ & $52,2 \pm 5,7$ & 0,80 \\
Menopausa (anos) & $47,8 \pm 4,3$ & $47,9 \pm 4,1$ & 0,20 \\
Tempo de menopausa (anos) & $4,7 \pm 5,1$ & $4,2 \pm 4,6$ & 0,65 \\
IMC (kg/ ${ }^{2}$ ) & $27,0 \pm 5,4$ & $26,3 \pm 4,3$ & 0,37 \\
\hline
\end{tabular}

"t de Student; IMC= índice de massa corpórea.

Na ultra-sonografia transvaginal não se observou variação nos valores da espessura endometrial nos Grupos Isoflavona e Placebo (0,28 $\mathrm{mm}$ versus $0,26 \mathrm{~mm}$, respectivamente), como mostra a Tabela 3 .

A Tabela 4 mostra a avaliação subjetiva da resposta terapêutica global em pacientes usuárias de isoflavona ou placebo após três meses de tratamento. Não houve diferença significativa entre os dois grupos.

Ao final do estudo, entre as usuárias da isoflavona, foram encontrados: desaparecimento completo dos fogachos em 26,2\% (11/42), melhora parcial em 38\% (16/42) e ausência de resposta em 35,8\% (15/42). No Grupo Placebo, observaram-se melhora total das ondas de calor em 18,7\% (9/48), melhora parcial em 39,6\% (19/48) e em 41,7\% (20/48) não houve alteração dos sintomas. Não houve diferença estatisticamente significativa entre os dois grupos, como mostra a Tabela 4.

\section{Discussão}

Os resultados deste ensaio clínico sugerem que a utilização de isoflavona de soja, em cápsulas manipuladas, na dosagem de $100 \mathrm{mg} / \mathrm{dia}$, por 12 semanas, não é mais efetiva que o placebo, para redução dos fogachos ou outros sintomas relacionados ao hipoestrogenismo em mulheres menopausadas.

Muitas mulheres têm usado a isoflavona para o alívio dos sintomas climatéricos, mas ainda não existem evidências científicas sobre a eficácia dessa opção terapêutica ${ }^{7}$. O efeito da suplementação dietética padronizada de soja tem sido estudado em alguns ensaios clínicos, que utilizaram doses semelhantes ou menores que a utilizada neste estudo por um período de 12 semanas. Estes estudos investigaram o efeito da suplementação diária de isoflavona e observaram redução estatisticamente significante na freqüência diária dos fogachos, comparado ao Grupo Controle ${ }^{15,16}$. Estudos controlados e meta-análise recentes também têm mostrado o efeito positivo de 50 a $100 \mathrm{mg} / \mathrm{dia}$ de isoflavona utilizada por três a 12 meses, na redução da freqüência dos fogachos ${ }^{8-10,17}$.
Tabela 2 - Índice de Kupperman (IK) e fogachos (medianas) nas pacientes usuárias de isoflavona ou placebo, antes e após três meses de tratamento.

\begin{tabular}{|c|c|c|c|}
\hline & $\begin{array}{l}\text { Isoflavona } \\
(n=42)\end{array}$ & $\begin{array}{l}\text { Placebo } \\
(n=48)\end{array}$ & $\mathbf{p l}^{*}$ \\
\hline \multicolumn{4}{|l|}{ IK } \\
\hline Antes do tratamento & 132 & 144 & 0,49 \\
\hline Após tratamento & 64 & 82 & 0,57 \\
\hline $\mathrm{p2}^{* *}$ & 0,46 & 0,16 & \\
\hline \multicolumn{4}{|l|}{ Fogachos } \\
\hline Antes do tratamento & 24 & 24 & 0,78 \\
\hline Após tratamento & 20 & 20 & 0,34 \\
\hline $\mathrm{p} 2^{* *}$ & 0,07 & 0,08 & \\
\hline
\end{tabular}

"pl=placebo versus isoflavona, antes e após tratamento (teste de Mann-Whitney); "* 2 =placebo antes versus após três meses de tratamento; isoflavona antes versus após três meses de tratamento (teste de Mann-Whitney).

Tabela 3 - Espessura endometrial avaliada pela medida do eco endometrial (mm), por meio da ultra-sonografia transvaginal, nas pacientes usuárias de isoflavona ou placebo,

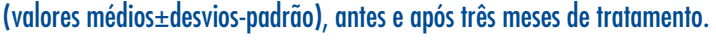

\begin{tabular}{lccc}
\hline Eco endometrial & $\begin{array}{c}\text { Isoflavona } \\
(\mathrm{n}=42)\end{array}$ & $\begin{array}{c}\text { Placebo } \\
(\mathrm{n}=48)\end{array}$ & $\mathrm{p}^{*}$ \\
\hline Eco antes do tratamento & $0,28 \pm 0,23$ & $0,26 \pm 0,21$ & 0,72 \\
\hline Eco após tratamento & $0,28 \pm 0,23$ & $0,26 \pm 0,21$ & 0,72 \\
\hline
\end{tabular}

"t de Student.

Tabela 4 - Avaliação subjetiva da resposta terapêutica global, em pacientes usuárias de isoflavona (número/\%) ou placebo, após três meses de tratamento.

\begin{tabular}{lcc}
\hline Resposta terapêutica & $\begin{array}{c}\text { Isoflavona } \\
(\mathrm{n}=42)\end{array}$ & $\begin{array}{c}\text { Placebo } \\
(\mathrm{n}=48)\end{array}$ \\
\hline Remissão tołal & $11(26,2)$ & $9(18,7)$ \\
Remissão parcial & $16(38,0)$ & $19(39,6)$ \\
Ausência de resposta & $15(35,8)$ & $20(41,7)$ \\
Total & $42(100)$ & $48(100)$ \\
\hline
\end{tabular}

$\chi^{2}=0,62 ; p=0,73$ (teste do $\chi^{2}$ ).

Por outro lado, outros autores, em estudo placebo controlado em mulheres na pós-menopausa que usaram 50 a $100 \mathrm{mg} /$ dia de isoflavona, não mostraram diferença significativa na redução da incidência de fogachos entre as pacientes após três a 12 meses de tratamento, sem benefícios adicionais do uso da isoflavona de soja em relação ao placebo, para o tratamento dos sintomas climatéricos ${ }^{18-20}$. Recentemente, outros estudos demonstraram que a isoflavona não é mais efetiva que o placebo no alívio dos sintomas vasomotores ${ }^{21,22}$. Metaanálise recente, envolvendo 17 estudos controlados, mostrou que os efeitos dos extratos de isoflavona sobre os sintomas climatéricos são inconsistentes ${ }^{7}$. Nossos resultados, embora não tenham mostrado redução da severidade dos fogachos ou outros sintomas climatéricos após 12 semanas de tratamento com isoflavona em 
comparação com o placebo, mostrou uma tendência à redução nos fogachos em cada um dos grupos, Placebo ou Isoflavona, após o tratamento, em comparação à avaliação basal. Resultados semelhantes foram observados por outros autores, que avaliaram pacientes previamente tratadas por câncer de mama, randomizadas para receber proteína de soja ou placebo, durante 12 semanas de tratamento. Observou-se que os dois grupos apresentaram significativa redução do número de fogachos, mas nenhuma diferença entre os grupos, sugerindo um efeito placebo da isoflavona ${ }^{23}$. Portanto, se uma intervenção medicamentosa for necessária e a terapia de reposição hormonal for contra-indicada ou indesejada, o uso dos inibidores seletivos da recaptação da serotonina tem sido sugerido ${ }^{7}$.

Embora vários fitoestrogênios tenham afinidade por receptores estrogênicos, a atividade específica no endométrio ainda não é conclusiva. Em nosso ensaio clínico, após 12 semanas de tratamento com a isoflavona, nenhum espessamento endometrial foi observado à avaliação ecográfica. Os ensaios clínicos que avaliaram os efeitos endometriais da suplementação de isoflavona em mulheres na pós-menopausa, por meio da avaliação ultra-sonog ráfica do endométrio, diferem em relação a dose e duração do tratamento; os resultados, porém, têm sido relativamente concordantes. A maioria desses estudos não observaram aumento da espessura ecográfica do endométrio com doses diárias de 45 a $110 \mathrm{mg}$ de isoflavona, por no mínimo 90 dias ${ }^{10-12,15,20}$. O uso de 70 a $100 \mathrm{mg}$ de isoflavona de soja parece não ter efeito na espessura endometrial ${ }^{20,24}$, no índice de pulsatilidade das artérias uterinas ${ }^{20}$, assim como parece não interferir na expressão dos marcadores de proliferação tecidual, o KI-67, sugerindo ausência de efeito proliferativo da isoflavona no endométrio após 90 dias de uso de $100 \mathrm{mg}$ desse fitoesteróide ${ }^{24}$. O uso da isoflavona, em longo prazo, em macacas cinomólogas ooforectomizadas e submetidas a uma dieta rica em isoflavona de soja parece não estimular a proliferação uterina ou mamária ${ }^{25}$. Alguns estudos epidemiológicos mostraram, nas populações que consomem alto teor de soja na dieta, menor risco para câncer endometrial, sem consenso em relação à quantidade de isoflavona consumida habitualmente por essas populações, estimando-se que seja entre 3 e $28 \mathrm{mg}$ por $\mathrm{dia}^{26}$.
Conseqüentemente, a importância de outros fatores que podem desempenhar um fator protetor ao câncer de endométrio deve ser considerada nessas populações. Um ensaio clínico randomizado e controlado avaliou o efeito de $150 \mathrm{mg}$ de isoflavona, utilizada durante cinco anos, nas características histológicas do endométrio em mulheres na pós-menopausa. Observaram que a atrofia endometrial foi o achado histológico mais freqüente, sem diferença significativa entre os grupos. No entanto, a incidência de hiperplasia $(3,4 \%)$ foi significativamente maior entre as usuárias de isoflavona ${ }^{3}$. Outros autores, embora não tenham observado alterações na espessura endometrial, detectaram algum grau de atividade mitótica endometrial, durante o uso da isoflavona em mulheres na pós-menopausa ${ }^{13}$. Além disso, um forte efeito estrogênico da isoflavona de soja foi demonstrado em ratas, tais como o aumento do volume uterino, queratinização do epitélio vaginal e metaplasia do epitélio escamoso uterino ${ }^{27}$.

É reconhecido o fato de que os fitoestrogênios podem comportar-se como um agonista ou antagonista estrogênico, dependendo da dose, duração do uso, afinidade a proteínas carreadoras, metabolismo individual, estado estrogênico intrínseco, assim como a distribuição tecidual dos subtipos de receptores estrogênicos, alfa ou beta ${ }^{4}$. Os alfa-receptores (RE- $\alpha$ ) são os principais receptores encontrados na mama e no útero, e os beta-receptores (RE- $\beta$ ), no osso e no sistema cardiovascular. O estradiol tem afinidade por ambos os receptores, enquanto as isoflavonas são sete vezes mais seletivas para os RE- $\beta$ do que para os RE- $\alpha 4$. Quando o tratamento for prolongado, ou quando for administrado a mulheres na pósmenopausa após longo período de hipoestrogenismo, a ação agonista pode ser mais evidente e um possível efeito estrogênico poderá ser visto em pacientes predispostas ${ }^{28}$. Evidentemente, todas essas possibilidades necessitam de confirmação e, portanto, de estudos adicionais sobre a farmacologia e os possíveis efeitos biológicos da isoflavona no endométrio, para que seu uso seja adequado e seguro na pós-menopausa.

Concluímos que a isoflavona de soja, na dose de $100 \mathrm{mg} / \mathrm{dia}$, por 12 semanas, não é mais efetiva que o placebo para a redução das ondas de calor e sintomas do hipoestrogenismo em mulheres na pós-menopausa, sem efeitos na espessura endometrial. 
1. Rossouw JE, Anderson GL, Prentice RL, LaCroix AZ, Kooperberg C, Stefanick ML, et al. Risks and benefits of estrogen plus progestin in health postmenopausal women: principal results from the Women's Health Initiative Randomized Controlled Trial. JAMA. 2002;288(3):321-33.

2. Huntley AL, Ernst E. A systematic review of herbal medicinal products for the treatment of menopausal symptoms. Menopause. 2003; 10(5):465-76.

3. Unfer V, Casini ML, Costabile L, Mignosa M, Gerli S, Di Renzo GC. Endometrial effects of long-term treatment with phytoestrogens: a randomized, double-blind, placebo-controlled study. Fertil Steril. 2004;82(1): 145-8.

4. Morito K, Aomori T, Hirose T, Kinjo J, Hasegawa J, Ogawa S, et al. Interaction of phytoestrogens with estrogen receptors alpha and beta (II). Biol Pharm Bull. 2002;25(1):48-52.

5. Nagata C, Takatsuka N, Kawakami N, Shimizu H. Soy product intake and hot flushes in Japanese women: results from a communitybased prospective study. Am J Epidemiol. 2001;153(8):790-3.

6. Albert A, Altabre C, Baró F, Buendía E, Cabero A, Cancelo M, et al. Efficacy and safety of a phytoestrogen preparation derived from Glycine max (L.) Merr in climacteric symptomatology: a multicentric, open, prospective, non-randomized trial. Phytomedicine. 2002;9(2):85-92.

7. Nelson HD, Vesco KK, Haney E, Fu R, Nedrow A, Miller J, et al. Nonhormonal therapies for menopausal hot flashes: systematic review and meta-analysis. JAMA. 2006;295(17):2057-71.

8. Drews K, Seremak-Mrozikiewicz A, Puk E, Kaluba-Skotarczak A, Malec M, Kazikowska A. The safety and tolerance of isoflavones (Soyfem) administration in postmenopausal women. Ginekol Pol. 2007;78(5):361-5.

9. Cheng G, Wilczek B, Warner M, Gustafsson JA, Landgren BM. Isoflavone treatment for acute menopausal symptoms. Menopause. 2007; 14(3 P+ 1):468-73.

10. Nahas EA, Nahas-Neto J, Orsatti FL, Carvalho EP, Oliveira ML, Dias R. Efficacy and safety of a soy isoflavone extract in postmenopausal women: a randomized, double-blind, and placebo-controlled study. Maturitas. 2007 Oct 1; [Epub ahead of print].

11. D'Anna R, Cannata ML, Atteritano M, Cancellieri F, Corrado F, Baviera $G$, et al. Effects of the phytoestrogen genistein on hot flushes, endometrium, and vaginal epithelium in postmenopausal women: a 1-year randomized, double-blind, placebo-controlled study. Menopause. 2007;14(4):648-55.

12. Kaari C, Haidar MA, Junior JM, Nunes MG, Quadros LG, Kemp $C$, et al. Randomized clinical trial comparing conjugated equine estrogens and isoflavones in postmenopausal women: a pilot study. Maturitas. 2006;53(1):49-58.

13. Wolff LPG, Martins MR, Bedone AJ, Monteiro IMU. Avaliação do endométrio em menopausadas após uso de isoflavonas. Rev Assoc Med Bras (1992). 2006;52(6):419-23.

14. Williamson-Hughes PS, Flickinger BD, Messina M, Empie MW. Isoflavone supplements containing predominantly genistein reduce hot flash symptoms: a critical review of published studies. Menopause. 2006;13(5):831-9.
15. Crisafulli A, Marini H, Bitto A, Altavilla D, Squadrito G, Romeo A, et al. Effects of genistein on hot flushes in early postmenopausal women: a randomized, double-blind EPT- and placebo-controlled study. Menopause. 2004; 11 (4):400-4.

16. Albertazzi P, Steel SA, Bottazzi M. Effect of pure genistein on bone markers and hot flushes. Climacteric. 2005;8(4):371-9.

17. Howes LG, Howes JB, Knight DC. Isoflavone therapy for menopausal flushes: a systematic review and meta-analysis. Maturitas. 2006;55(3):203-11

18. Verhoeven $\mathrm{MO}$, van der Mooren $\mathrm{M}$, van de Weijer $\mathrm{PH}$, Verdegem PJ, van der Burgt LM, Kenemans $P$, et al. Effect of a combination of isoflavones and Actaea racemosa Linnaeus on climacteric symptoms in healthy symptomatic perimenopausal women: a 12-week randomized, placebo-controlled, double-blind study. Menopause. 2005; 12(4):412-20.

19. Kok L, Kreijkamp-Kaspers S, Grobbee DE, Lampe JW, van der Schouw YT. A randomized, placebo-controlled trial on the effects of soy protein containing isoflavones on quality of life in postmenopausal women. Menopause. 2005; 12(1):56-62.

20. Penotti M, Fabio E, Modena AB, Rinaldi M, Omodei U, Viganó $P$. Effect of soy-derived isoflavones on hot flushes, endometrial thickness, and the pulsatility index of the uterine and cerebral arteries. Fertil Steril. 2003;79(5):1112-7.

21. Lewis JE, Nickell LA, Thompson LU, Szalai JP, Kiss A, Hilditch JR. A randomized controlled trial of the effect of dietary soy and flaxseed muffins on quality of life and hot flashes during menopause. Menopause. 2006;13(4):631-42.

22. MacGregor CA, Canney PA, Patterson G, McDonald R, Paul $J$. A randomised double-blind controlled trial of oral soy supplements versus placebo for treatment of menopausal symptoms in patients with early breast cancer. Eur J Cancer. 2005;41(5):708-14.

23. Van Patten CL, Olivotto IA, Chambers GK, Gelmon KA, Hislop TG, Templeton E, et al. Effect of soy phytoestrogens on hot flushes in postmenopausal women with breast cancer: a randomized controlled trial. J Clin Oncol. 2002;20(6):1449-55.

24. Nikander $E$, Rutanen $E M$, Nieminen $P$, Wahlström T, Ylikorkala $O$, Tiitinen A. Lack of effect of isoflavonoids on the vagina and endometrium in postmenopausal women. Fertil Steril. 2005;83(1):137-42.

25. Wood CE, Register TC, Anthony MS, Kock ND, Cline JM. Breast and uterine effects of soy isoflavones and conjugated equine estrogens in postmenopausal female monkeys. J Clin Endocrinol Metab. 2004;89(7):3462-8.

26. Nakamura Y, Tsuji S, Tonogai Y. Determination of the levels of isoflavonoids in soybeans and soy-derived foods and estimation of isoflavonoids in Japanese daily intake. J AOAC Int. 2000;83(3):635-50.

27. Cline JM, Franke AA, Register TC, Golden DL, Adams MR. Effects of dietary isoflavone aglycones on the reproductive tract of male and female mice. Toxicol Pathol. 2004;32(1):91-9.

28. Wuttke W, Jarry H, Seidlová-Wuttke D. Isoflavones: safe food additives or dangerous drugs? Ageing Res Rev. 2007;6(2):150-88. 